\title{
757.
}

\section{ON A SMITH'S PRIZE QUESTION, RELATING TO POTENTIALS.}

[From the Messenger of Mathematics, vol. xI. (1882), pp. 15-18.]

A SPHERICAL shell is divided by a plane into two segments $A$ and $B$, one of them so small that it may be regarded as a plane disk: trace the curves which exhibit the potentials of the two segments and of the whole shell respectively, in regard to a point $P$ moving along the axis of symmetry of the two segments.

Criticise the following argument:

The potential of the segment $A$ in regard to a point $P$, coordinates $(x, y, z)$, is one and the same function of $(x, y, z)$ whatever be the position of $P$; similarly the potential of the segment $B$ in regard to the same point $P$ is one and the same function of $(x, y, z)$ whatever be the position of $P$ : hence the potential of the whole shell in regard to the point $P$ is one and the same function of $(x, y, z)$ whatever be the position of $P$.

The question is taken from my memoir "On Prepotentials," Phil. Trans. vol. 165 (1875), pp. 675-774, [607]; and the figure of the curves is given p. 689*. There is no difficulty in tracing them by means of the expression for the potential of a plane circular disk in regard to a point on its axis of symmetry: it was in order that they might be so traced, that one of the segments was taken to be small; but I had overlooked the circumstance that the formula for the disk is in fact only a particular case of a similar and equally simple formula for the spherical segment: viz. (as was found in one of the papers) the potential of a spherical segment in regard to a point on the axis is $=\frac{2 \pi a}{\rho}\left(\rho_{1} \sim \rho_{2}\right)$, where $\rho, \rho_{1}, \rho_{2}$ are the distances of the attracted point from the centre of the sphere and from the centre and the circumference respectively of the segment. The segments might therefore just as well have been any two segments whatever, or (to take the most symmetrical case) they might have been hemispheres.

As to the argument: the assertion in regard to the potential of the segment

[* This Collection, vol. Ix. p. 333.] 
$A$ is based upon the consideration of this segment alone; and, on the ground that we can without crossing the segment pass from any one position of $P$ to any other position of $P$, it is inferred that the potential is one and the same function of the coordinates, whatever be the position of $P$ : it is therefore unassailable by any considerations in relation to the non-existent segment $B$. Similarly the assertion in regard to the potential of the segment $B$ is based upon the consideration of this segment alone, and it is unassailable upon any considerations in regard to the nonexistent segment $A$ : the potential of the whole sphere is certainly the sum of the potentials of the segments $A$ and $B$ : it is therefore altogether off the purpose to object that in the case of the whole sphere we cannot pass from a point outside the sphere to a point inside the sphere without crossing one or other of the segments $A$ and $B$. I consider that the two assertions are each of them true, and that the conclusion is a legitimate one, but it is true only in the sense in which $a+x+\sqrt{ }\left[(a-x)^{2}\right]$ is one and the same function of $x$ whatever be the value of $x$ : this is so, if $\sqrt{ }\left[(a-x)^{2}\right]$ denotes indifferently or successively the two functions $\pm(a-x)$ : but if, $a$ and $x$ being real, $\sqrt{ }\left[(a-x)^{2}\right]$ is taken to mean the positive value, then the function $a+x+\sqrt{ }\left[(a-x)^{2}\right]$ is $=2 a$ or $=2 x$ according as $a-x$ is positive or negative.

Fig. 1.

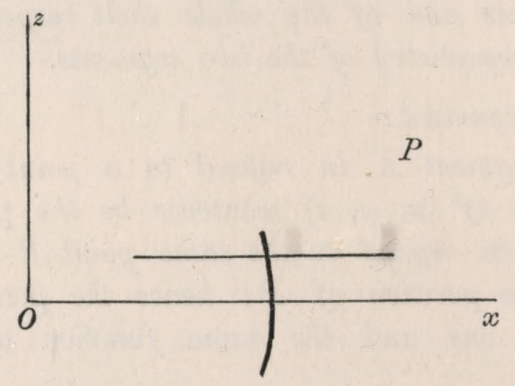

In further illustration, let the dark line of fig. 1 represent the intersection of an unclosed surface, or segment, by the plane of $x z$ taken to be that of the paper, and consider the potential of the segment in regard to a point $P$ in the plane of the paper, coordinates $x, z$. We have the potential $V$ defined as a function of $x, z$ by an equation $V=a$ definite integral, depending on the parameters $x, z$, and being in general a transcendental function of $(x, z) ; V$ is a real, one-valued, finite, continuous function of $x, z$ : in particular, if the point $P$, moving in any manner, traverses the dark line, there is not any discontinuity in the value of $V$. There is however in this case a discontinuity in the differential coefficients of $V$ : if to fix the ideas we imagine $P$ moving parallel to the axis of $x$, so that $z$ is taken to be constant and $V$ a function of $x$ only, then when the path of $P$ crosses the black line there is in general an abrupt change of value in $\frac{d V}{d x}$. Taking $V$ as a coordinate $y$ at right angles to the plane of the paper, a section by any plane parallel to that of $x y$ is (when the trace of the plane upon that of $x z$ does not meet the dark line) a continuous curve; but when the trace meets the dark line, then for this value of $x$ there is an abrupt change of direction in the section. 
If (as may very well happen in particular cases) $V$ is algebraically determinable, then, $q u \hat{a}$ one-valued function of $(x, z), V$ is not any root $y$ at pleasure of an algebraical equation $\phi(x, y, z)=0$, but it is for any given values of $(x, z)$, some one determinate root $y_{1}$ of this equation: and we thus see how in this case the beforementioned discontinuity in the value of $\frac{d V}{d x}$ must arise: viz. when the trace of the plane meets the dark line the section is a curve having a double point; and, for the positions of $P$ on the two sides of the dark line, we have $V$ the ordinate belonging to different branches of the curve of section. If the path of $P$ passes through an extremity of the dark line, then the curve of section will, instead of a double point, have in general a cusp; and when the path of $P$ does not cross the dark line, then the curve of section is a continuous line without singularity. It may be added that the surface $\phi(x, y, z)=0$ must have a nodal line which as to a certain finite portion thereof is crunodal, giving the before-mentioned double points of the sections, but as to the residue thereof is acnodal or isolated.

It may happen that (the surface being algebraical) any particular section thereof, instead of being a single curve having a double point as above, breaks up into two distinct curves, so that for the two positions of $P$, we have $V$ the ordinate of two distinct curves: and this is what really happens in the case of $P$ a point on the axis of a circular disk or a spherical segment: thus in the case of the disk, taking $c$ for the radius, and $x$ for the distance from the centre of the disk, the formula is $V=2 \pi\left\{\sqrt{ }\left(c^{2}+x^{2}\right) \pm x\right\}$; or writing $V \div 2 \pi=y$, the section is made up of the two distinct hyperbolas $y(y-2 x)=c^{2}$, and $y(y+2 x)=c^{2}$.

It may be remarked that in each case, it is only for $P$ on the axis that the potential is algebraical.

In the case of the hemispheres, drawing $O M$ a radius at right angles to the axis, the formula for the potential of an axial point $P$ is of the form

$$
V=\frac{2 \pi \cdot O M}{O P}(P M \sim P A),
$$

or writing $V=2 \pi y$ we have for the hemisphere $A$, the curve (1) or (2) according as $(x-a)$ is positive or negative; and for the hemisphere $B$ the curve (3) or (4) according as $x+a$ is positive or negative; viz. the equations are
(1) $y=\frac{a}{x}\left\{\sqrt{ }\left(a^{2}+x^{2}\right)-(x-a)\right\}$,
(2) $y=\frac{a}{x}\left\{\sqrt{ }\left(a^{2}+x^{2}\right)+(x-a)\right\}$,
(3) $y=\frac{a}{x}\left\{\sqrt{ }\left(a^{2}+x^{2}\right)-(x+a)\right\}$,
(4) $y=\frac{a}{x}\left\{\sqrt{ }\left(a^{2}+x^{2}\right)+(x+a)\right\}$, 
being four cubic curves. The whole curve (1) is shown in fig. 2 , and the others are Fig. 2.

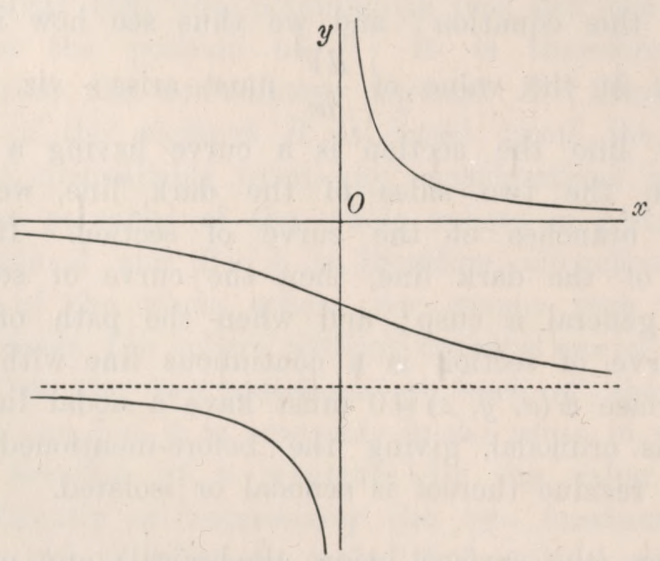

equal or opposite curves: the rationalised equation of (1) is in fact

$$
x=\frac{2 a^{2}(y+a)}{(y+a)^{2}-a^{2}},
$$

and by writing $-a$ for $a$, and in each equation $-x$ for $x$, we have the rational equations of the other three curves.

But, drawing only the required portions of the curves, we have fig. 3 exhibiting

Fig. 3.

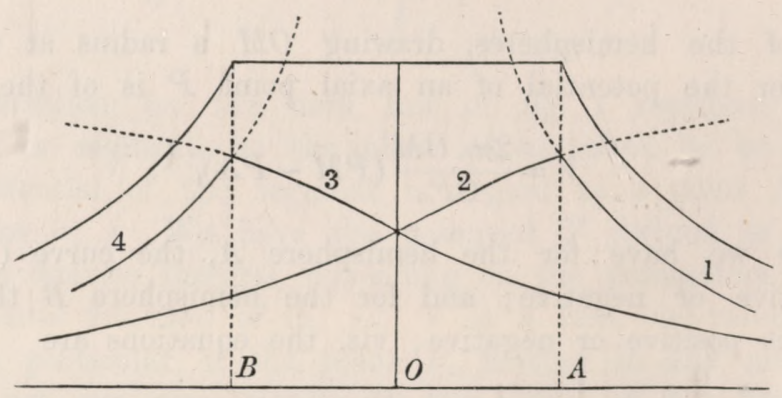

the potentials of the two hemispheres $A$ and $B$; and also the discontinuous potential of the whole shell, the ordinate for this last being the sum of the ordinates for the two hemispheres respectively. 